\title{
Lymph node macrophages: drug-related reaction or infectious-lesion?
}

\author{
Adriana Handra-Luca ${ }^{1,2}$ \\ ${ }^{1}$ Department of Pathology, APHP GHU Avicenne, Bobigny, ${ }^{2}$ UFR SMBH Université Paris Nord, Bobigny, France
}

The involvement of myeloid-derived cells in IBD pathogenesis both in gut inflammatory loci and lymphoid organs is well known, as reviewed by Kim et al. ${ }^{1}$ We had recently the opportunity to identify groups of macrophages in a lymph node detected on a cholecystectomy specimen. The patient (man, 47 years) was admitted for abdominal pain, hyperleukocytosis and elevated CRP. The medical history revealed $\mathrm{CD}$ (diagnosed at the age of 33 years) with recurrent anal fissures (treated by 5 surgeries). The medical treatment comprised initially mesalazine and more recently ifliximab and azathioprine, azathioprine being associated with abdominal pain. The CT-scan at the time of admission showed a lithiasic gallbladder with thickened wall. A coelioscopic cholecystectomy was performed. The resected specimen consisting in 2 fragments measuring 7.4 and $4.4 \mathrm{~cm}$. The microscopy diagnosis was that of subacute cholecystitis with foci of intestinal metaplasia. The lymph node detected on the surgical specimen, contained several extravascular macrophages, PAS and CD68 positive (Fig. 1). The immunohistochemistry for the Tropheryma whipplei was negative.

Extravascular macrophage accumulations in lymph nodes are rare. ${ }^{2}$ Transient accumulations are reported in rhesus monkey models, during acute SIVmac239 virus infection. ${ }^{3}$ In the case we report, the histogenesis of the lymph node macrophage accumulation is difficult to precise. An infectious origin was ruled out as based on the medical history and negativity of $T$. whipplei immunohistochemistry. Remained the hypothesis of drug-related lesions, both ifliximab and
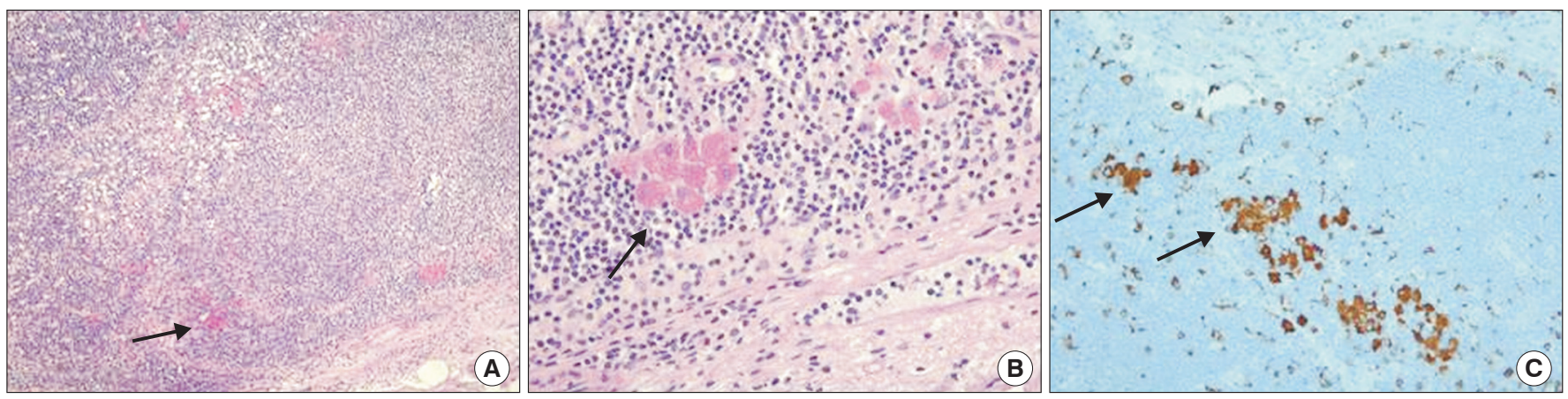

Fig. 1. The gallbladder lymph node contained several extravascular PAS- $(A, B, H \& E)$ and CD68-positive macrophages (C, immunohistochemical staining) (arrows). Original magnification $\times 2.5(A), \times 40(B)$, and $\times 20(C)$. 
azathioprine being reported to influence the macrophage number. While the impact of ifliximab in CD is varied, reported to result both in CD68-positive macrophage reduction $^{4}$ and in regulatory macrophage induction, ${ }^{5}$ including with occurrence of a macrophage activating syndrome, ${ }^{6}$ azathioprine is reported to affect the number of peritoneal macrophages in mice models, when administered at high dose and over a long period. ${ }^{7}$ However, to note would also be the possibility of a pre-granulomatous, macrophage reaction, as related to influx of IBD myeloid-derived cells, ${ }^{1}$ associated or not to the potential impact of the medical treatment.

In conclusion, macrophage accumulations may occur in the gallbladder lymph node in the context of IBD, possibly as related to medical treatments with infliximab and/or azathioprine.

\section{FINANCIAL SUPPORT}

The authors received no financial support for the research, authorship, and/or publication of this article.

\section{CONFLICT OF INTEREST}

No potential conflict of interest relevant to this article was reported.

\section{AUTHOR CONTRIBUTION}

Writing and approval of final manuscript: Adriana HandraLuca.

\section{REFERENCES}

1. Kim YJ, Chang SY, Ko HJ. Myeloid-derived suppressor cells in inflammatory bowel disease. Intest Res 2015;13:105-111.

2. Rosai J. Rosai and Ackerman's surgical pathology. 10th ed. Philadelphia: Elsevier Mosby, 2011.

3. Otani I, Mori K, Sata T, et al. Accumulation of MAC387+ macrophages in paracortical areas of lymph nodes in rhesus monkeys acutely infected with simian immunodeficiency virus. Microbes Infect 1999;1:977-985.

4. Caprioli F, Bosè F, Rossi RL, et al. Reduction of CD68+ macrophages and decreased IL-17 expression in intestinal mucosa of patients with inflammatory bowel disease strongly correlate with endoscopic response and mucosal healing following infliximab therapy. Inflamm Bowel Dis 2013;19:729-739.

5. Vos AC, Wildenberg ME, Arijs I, et al. Regulatory macrophages induced by infliximab are involved in healing in vivo and in vitro. Inflamm Bowel Dis 2012;18:401-408.

6. Chauveau E, Terrier F, Casassus-Buihle D, Moncoucy X, Oddes B. Macrophage activation syndrome after treatment with infliximab for fistulated Crohn's disease. Presse Med 2005;34:583584.

7. Gassmann AE, van Furth R. The effect of azathioprine (Imuran) on the kinetics of monocytes and macrophages during the normal steady state and an acute inflammatory reaction. Blood 1975;46:51-64. 